mischen Eigenschaften des Th, ist ein $\beta$-Strahler und bat eine Halbwertszeit von 25,5 - 0,5 Stunden.

Radium. Úber die Extrahierung des Radiums aus den Erzen von Olary (Australien) s. Chem. Zentralbl. 1914, I, 1990. Methoden zur Anreicherung arbeiteten aus F. U l z e r und R. So m m e r (D. R. P. $263330, \mathrm{Kl} .12 \mathrm{~m}$ vom 17./4. 1913), K u n h e i m \& Co., D. R. P. 264901 , Kl. $12 m$, 15./6. 1912, ferner E. E b le r und W. B end e r durch fraktionierte Adsorption und Desadsorption von $\mathrm{Ra}-\mathrm{Ba}$ Sulfaten an Mangansuperoxydhydrat (Zeitschr. f. anorgan. Chem. 84, 77, sowie E. E b l e r D. R. P. 267071 , Kl. $12 m$ vom 12./12. 1912). Utber die Aufarbeitung von Ra-Meso-Thhaltigen Gemischen s. E b l e r, D. R. P. $278260, \mathrm{Kl} .12 \mathrm{~m}$ vom 18./9. 1913. Ich verzichte hier mit Rücksicht auf dic zusammenfassende Arbeit von $\mathrm{E}$. $\mathrm{E}$ b l e r in dieser Z:itschrift ${ }^{64}$ ) auf eingehendere Beschreibungen. Auch die quantitative Bestimmung des Radiums vo $\mathrm{n} \mathrm{Ebler}$ nach der Emanationsmethode ist in dieser Zeitschrift bereits gesondert behandelt ${ }^{65}$ ).

Von der durchdringenden Strahlung des Radiums wiesen Frau $P$. Curie und K a merling On es nach, daß sie beim Siedepunkt des Wasserstoffs $\left(20,3^{\circ}\right.$ absol.), wenn überhaupt, sich um 1/1000 von der bei gewöhnlicher Temperatur unterscheidet.

Uber die $\mathrm{R}$ a di umemanation ist nichts wesentlich Neues $z u$ berichten. In einem zusammenfassenden Aufsatz ${ }^{66}$ ) hat G. v. $\mathrm{H}$ e ve s y dic wichtigsten Eigenschaften der Emanationen nach den neusten Forschungen zusammengestellt. Außer den in der Tabelle gegebenen Eigenschaften sind hier für Radiumemanation mitgeteilt: Ihr Volumen im radioaktiven Gleichgewicht mit $1 \mathrm{~g} \mathrm{Ra}$ $=0,6 \mathrm{cmm}$, ihre Dichte $(\mathrm{H}=: 1) \mathrm{zu} 11 \mathrm{l}$, die Dichte der flüssigen Emanation bcim Siedepunkt $5-6$, Siedepunkt $-65^{\circ}$, kritischer Punkt $+104,5^{\circ}$, Gefricrpunkt $-71^{\circ}$ Beim RaA wies F l e $\mathrm{c} \mathrm{k}^{67}$ ) nach, daß es chemisch identisch mit Polonium ist.

Eine Reibe von Arbeiten $R u$ therfor $\mathrm{ds}^{68}$ ) und sciner Schüler beschäftigte sich mit der $\beta$ - und, Strahlung von $\mathrm{RaB}$ und $\mathrm{RaC}$. Dic Analyse der $\beta$-Strahlen ergab bei RaB 16 verschiedene $\hat{\beta}$-Strahlengruppen, beim $\mathrm{RaC}$ ca. 50, die der $\gamma$-Strahlen lieferte bci RaB Anzeichen für mindestens zwei Gruppen, von denen eine sehr weich, dic andere recht durchdringend ist. Die $\gamma$-Strahlen von $\mathrm{RaC}$ bestehen im wesentlichen aus einem Typus, der in Aluminium exponential absorbicrt wird. Interessant ist dic Beobachtung, daß die $\gamma$-Strahlen von $\mathrm{RaB}$ und $\mathrm{RaC}$ beim Durchgang durch Materie Anlaß zur Entstehung von $\beta$-Strahlen sehr hohor Geschwindigkeit geben, die völlig verschieden sind von den primären $\beta$-Strahlen, die diese Radioclemente aussenden. Die Spektren der $;$-Strahlen von $\mathrm{RaB}$ und $\mathrm{RaC}$ gaben Veranlassung zu theoretisch interessanten Frörterungen.

Daß F. P a n e th und G. v. He ve s y mit allen Mitteln erfolglos versucht hatten, RaD von Blei zu trennen, ist bereits erwähnt worden. $\mathrm{Ch}$ a r les $\mathrm{S} t$ a e h l in g widerleyt denn auch einen von $H$ of $m$ a n $n$ und $W$ öl $l$ beschriebenen Versuch, $\operatorname{RaD}$ von $\mathrm{Pb}$ mit $\mathrm{Gr}$ ignards Reagens abzutrennen.

Vom RaE zeigte F. Pa n e $t h, d a ß$ es in saurer Lösung leicht diffundiert, schwerer in neutraler und ammoniakalischer Lösung.

RaF (Polonium). Wichtige Erfahrungen über die $\mathrm{A} \mathrm{b}$ scheidung dos Poloniums wurden im Wiener Institut für Radiumforschung durch F. P a n eth und G. v. He ves y gemacht ${ }^{69}$. Man hat bekanntlich das Polonium im Radioblei. Will man es daraus abscheiden, so verwendet man wegen des ausgeprägt metallischen, stark elektronegativen Charakters des Poloniums am besten die elektrolytische Abscheidung. Man stellt sich

64) Angew. Chem. 28, I, 25, 11 [1915]

65) Angew. Chem. 26, I, 658 [1913]

66) Jahrb. d. Radioakt. u. Elektronik 10. 198ff. [1913]

6i) .T. Chem. Soc. $103,1052$.

68) Phil.Mag. 25, 722; 26, 717, 937; 27, 854; 28, 263.

69) Sitzungsber. d. Wiener Akademic 121, 2194 [1912]; 122, 1049 [1913]: Wiener Monatshefte 19I5. 45. eine heiß gesättigte Lösung von Radiobleinitrat her, läßt auskrystallisieren, trennt die Krystallmasse von der Mutterlauge durch Zentrifugieren, verdünnt die Mutterlauge etwas, um weiteres Auskrystallisicren zu verhindern, und elektrolysiert darauf mit schwachem Strom.

(Fortsetzung folgt.)

\section{Die Gewinnung des Gheddawachses und das Wachs aus dem Wikingerschiff zu Oseberg.}

\author{
Von Haxis Fischer. \\ (Eingeg. 11./5. 1915.)
}

Herr Prof. S e be li e $n$ hat vor einiger'Zeit an dieser Stelle $^{1}$ ) eine sehr interessante Arbeit über ein Wachs, das in einem Wikingerschiffe in $\mathrm{O}$ s e berg gefunden wurde, veröffentlicht. John Sebelie $\mathbf{J}$ spricht an dieser Stelle den Wunsch aus, näheres über die Gewinnung des Bienenwachses in Ostindien zu erfahren. Da ich mich persönlich dort längere Zeit mit dem Studium des Gheddawachses befaßt habe ${ }^{2}$, möge mir gestattet scin, meine Erfahrungen hier zusammenfassend wicderzugeben. Die Arbeit John Sebeliens ist um so wertvoller für mich, als ich den Standpunkt vertrete, daß anormale Bienenwachse, abgesehen von dem gebleichten und ostindischen Wachse, nicht vorkommen ${ }^{3}$ ). Deswegen ist seine Beobachtung sehr wertvoll, daß durch gewisse Formen des Umschmelzens Verringerung der Säurezahl erzielt werden kann. Leider habe ich in Indien diesem Punkte weniger Aufmerksamkeit geschenkt, doch glaube ich nicht, daß die Ermiedrigung der Säurezahl des ostindischen und auch chinesischen Wachses durch die Umschmelzung hervorgerufen wird. Im folgenden mögen nun die verschiedenen Formen des Einsammelns und Umschmelzens angeführt werden. Es handelt sich dabei ausschließlich um das Wachs wilder Bienen, Apis dorsata, Apis florea, Apis in. di c a und der $\mathrm{Tr}$ ig on $\mathrm{c}$ - und $\mathrm{M}$ eli ponenarten.

Trotz der großen Ausdehnung Indiens, die etwa dem Flächeninhalt Furopas, ohne Rußland, gleichkommt, und trotz der immerhin beschränkten Verkehrsmittel wirdł'die Gewinnung des Bienenwachses in fast unveränderlicher Form von allen den . Djangelbewohnern ${ }^{4}$ ) ausgeführt, die Honig und Waben von Bäumen oder Felsen sammeln.

Die für die Wachsernte ergiebigsten und gebräuchlich. sten Zeiten sind die heißen Monate von Anfang April bis Mitte Juni.

Die Gewinnung der Waben geschieht überall in Indien nach nahezu derselben Methode. Diese besteht darin, daß zwei oder mehr Leute, die sich durch Einhüllen in Decken zunächst gegen die Bienen schützen, mit Hilfe von Feuer und Qualm die Insekten vertreiben und dann die Waben sammeln. Oft auch fertigen die Sammler aus Gras, trockenen Blättem und vermulmtem Holz Qualmfackeln, ersteigen damit die Bäume und bemächtigen sich, nachdem die Bienen durch den Rauch vertrieben wurden, der Stöcke. In Chotanagpur wird dieses Verfahren noch dadurch vereinfacht, daß die Sammler die Bäume, auf denen sich Bienensiedelungen befinden, umschlagen und dann zur Einsammlung der Waben schreiten. In anderen Teilen Indiens wieder geben sich die Eingeborenen, ehe sie ihren Sammelberuf ausführen, ein geheimnisvolles Ausseben, indem sie bestimmte Regeln in bezug auf ihre Lebensweise befolgen. Wegen der Gefahr, von wilden Tieren angefallen zu werden, wird meist am Tage gesammelt. In sichcren Gegenden gehen die Eingeborenen auch nachts auf Suche, da erfahrungsgemäß die Biene dann weniger sticht. In Behar wird zwar auch nur des Nachts gesammelt, doch benutzen die

1) Angow. Chem. 26, I, 689-692 [1913].

2) Z. öff. Chem. 19.147-158, 170-175, 188-195 [1913]; 20, 313 bis $315,409-419$ [1914]; 21, 17-25, 53-54 [1915]. Pharm. Ztg. 51, $1017-1019$ [1914]

3) Es wäre sehr interessant, zu erfahren, ob die norwegischen Wachse, die Herr Sebelien untersuchte, aus Stöcken stammen, in denen künstliche Mittelwaben zur Anwendung gekommen sind.

4) Es sei hier ausdrücklich hervorgehoben, daß die Schreibweise „Djangel" das indische Wort tatsächlich wiødergibt, während „Dschungel" oder „Djungel" nur eine englische Verballhornung ist. 
Eingeborenen noch besondere Schutzmaßregeln. Sie reiben sich nämlich mit einer aus Kräutern bereiteten Salbe ein, deren Zusammensetzung nur einem bekannt ist, und der seine Vorschrift nur in seiner Familie weiter vererbt, da sie eine sichere Einnahmequelle bietet. Dieses Mittel soll stichsicher machen. Nicht immer befinden sich die Waben auf Bäumen, sondern auch an überhängenden Felsenstürzen, deren Besteigung unmöglich ist. Hier nun erklimmen die Eingeborenen den Berg, an dem sich der Abhang befindet. Der Mutigste von ihnen läßt sich dann an Stricken in einem Netz oder Korbe herab, die Fackel im Arm, die hier meist der besseren Haltbarkeit wegen aus Mulm und $\mathrm{f} \mathrm{i}$ $\mathrm{s}$ c h e n Blättern besteht, und sucht nach Vertreibung der Bienen der Waben habhaft zu werden. Von besonderem Interesse dürften auch die Veranstaltungen sein, welche die Eingeborenen in Oberinindwin bei Beginn der Blüte treffen. Sie bauen in der Nähe ihrer Dörfer aus Bambusen Bienenstöcke. Nachdem sie ihren Waldgöttern geopfert haben, laden sie die Bienen unter bestimmten Gesängen zum Schwärmen ein, während dessen dazu ausersehene Männer mit Stäben, die aus getrockneten Fischen hergestellt sind, gegen Schildkrötenplatten schlagen. Dann verlassen sie die Stöcke, besuchen sie aber von Tag zu Tag, um zu sehen, ob Bienen geschwärmt haben, und befolgen jedesmal beim Anblick eines Schwarmes von neuem ihre Zeremonie. Hier gestaltet sich dann das Einsammeln der Waben ein facher, da es nur notwendig ist, durch Qualm die Bienen zu vertreiben.

Was nun die Waben selbst anlangt, so schwankt der Durchmesser von anderthalb Meter bis zur Größe einer Hand. Abgesehen von Godavari, wo man, um besseres reineres Wachs zu erhalten, den unteren schmutzigen, die Larven enthaltenen Teil abtrennt, wird ausschließlich die ganze Wabe eingeschmolzen. Selbstredend wird zunächst der Honig entfernt. Dies geschieht durch Ausquetschen mittels der Hand oder durch Eindrehen der Wabe in ein Stück Zeug. Dann wird die Wabe mit kaltem Wasser gewaschen, $\mathrm{um}$ die löslichen Stoffe zu entfermen, um dann in einem messingenen, eisernen oder tönemen Gefäß ganz wie in Europa umgeschmolzen, und sollte es notwendig sein, durch ein Stück Kleiderstoff filtriert, um in einem mit kaltem Wasser gefüllten Gefäß aufgefangen zu werden.

In einigen Gegenden wendet man besondere Methoden zur Reinigung und Klärung des Wachses an. So bringt man es in Betul, in den Zentralprovinzen, eine Nacht lang in ein Gefäß mit flüssigem Kuhdung. Am nächsten Tage wird es gewaschen, über Wasser geschmolzen und mittels eines Stückes Zeug von den groben Unreinlichkeiten getrennt. Durch diese Handhabung soll das Wachs sehr klar werden. Eine andere Art besonderer Reinigung wird in Nellore im Bezirk Madras vorgenommen. In das geschmolzene Wachs wird eine Hand voll Tamarindenblätter hineingeworfen, und die Eingeborenen versichern, daß hierdurch alle Fremdstoffe sich absetzen, und ein außerordentlich reines Wachs erhalten wird. Manchmal wird auch Salz dem Umschmelzwasser beigegeben, doch kann man sagen, daß andere Chemikalien nicht verwendet werden, ausgenommen Borax, der auch ein gutes Klärmittel bildet. In einem Teil des Panjabs gibt man, um das Wachs plastischer zu machen dem durchgeseihten und abgekühlten Wachs etwa ein Achtel seines Gewichts an Sesamöl hinzu, ehe es für den Markt fertiggestellt wird. Eine Beigabe aber, die in ganz Indien als allgemein verbreitet gelten darf, bildet die gepulverte Curcumawurzel (Curcuma longa) mit deren Hilfe man dem gereinigten Wachs eine goldgelbe künstliche Farbe gibt. Es sei hier noch auf eine Umschmelzmethode hingewiesen, die besonders in Hinterindien in der Kathagegend, in Birma, im Gebrauch ist. Starke Bambusrohre werden so zerschnitten, daß jedes Stück einseitig geschlossen ist. In jede dieser Röhren bringt man etwas Wasser, tut darauf eine Lage feinzersplitterter Bambusfaser und dann das Wachs. Das Ganze wird dann aufs Feuer gebracht. Der sich entwickelnde Dampf bringt das Wachs zum Schmelzen, die aus den Fasern ausgetriebene Luft entlüftet das Wachs und fördert das Absetzen der Verunreinigungen. Nachdem fast alles Wasser entwichen ist, wird das Ganze abgekühlt, und der Bambus aufgespalten. Der erhaltene Block reinen Wachses ist fertig für den Handel. Die Form, in der das Wachs ausgeführt wird, schwankt zwischen Kuchen-, Schüssel-, Zylinder-, Kugel- oder Blockform. Die Form für Blöcke bilden in den Boden gegrabene und mit flüssigem Kuhdung ausgeschmierte Löcher, die man besonders in Bombay und Madras häufig antrifft.

Nach dem eben Gesagten scheint es also nicht wahrscheinlich, daß die starke Verminderung der Säurezahl von 18-20 des europäischen Wachses durch die Umschmelzung auf 6-9 des indischen Wachses herabgedrückt werden kann. Meine eigenen Untersuchungen, die mit allerdings schon immer über Wasser umgeschmolzenem, indischen Wachs angestellt wurden, ergaben niemals eine Säurezahl, die derjenigen des europäischen Wachses glich (a b g e s ehen von Trigonenwachs). Es wird mir aber voraussichtlich nach Beendigung des Krieges möglich sein, Originalwaben zu erhalten, und ich werde dann Gelegenheit nehmen, auf deren Analysen zurückzukommen. Jedenfalls möchte ich nicht unerwähnt lassen, daß die Möglichkeit vorhanden ist, ein Wachs aus indischem und europäischem Wachs herzustellen, das den Zahlen des Wikingerwachses, die folgendermaßen lauteten:

\author{
Säurezahl: 16,6, \\ Esterzahl: 78,74, \\ Verseifungszahl: 95,30 , \\ Verhältniszahl : 4,74 ,
}

auch was die Jodzahl von 6,08 anlangt, entspricht. Für den vorliegenden Fall allerdings dürfte eine solche Mischung wohl nicht in Frage kommen. Denn Herr Prof. S e be l i e n hat gezeigt, daß die Wachssäure ein verbältnismäßig flüchtiger Körper ist; deswegen darf es meines Erachtens nicht wundernehmen, daß der niedrige Wert der Säurezahl des Wikingerwachses, der auf 16,6 herabgegangen ist, durch ein fast tausendjähriges Alter bedingt wurde.

Zum Schluß möchte ich nicht unerwähnt lassen, daß die von John Sebeli en als Kriterium angeführte gelbe Farbe keine typische Eigenschaft des Bienenwachses ist. In Indien zum Beispiel finden sich Wachse von grüngrauer, weißlichgrüner und sandgrauer Farbe, die aber mit Hilfe von Curcuma sehr schön gegelbt werden können. [A. 57.]

\section{Die Zusammensetzung des Palmkern- und Cocosnußöles.}

Von A. Heidúschisa.

(Eingeg. 21./5. 1915:;

Utber die Zusammensetzung des Palmkern- und Cocosnußöls macht G. D. E ls d o $n^{1}$ ) neuerdings in einer kurzen Veröffentlichung Angaben, deren Richtigkeit nicht ohne weiters einleuchtend sind. So stimmt z. B. der Gehalt von Ölsäure (Palmkernöl 4, Cocosnußöl 2) keineswegs mit der Jodzahl dieser Fette überein, auch ist der immerhin hohe Gehalt beider Fette an Palmitinsäure und Stearinsäure auffällig. Zahlreiche Untersuchungen des Palmkern- und Cocosnußöles des Handels von mir ${ }^{2}$ ) und meinen Mitarbeitern haben nie die Anwesenheit dieser Fettsäuren in wesentlicher Menge ergeben, und man kann annehmen, daß in den für Speisezwecke in Betracht kommenden Fetten dieser Art höchstens Spuren von Palmitin- und Stearinsäure vorhanden sind. Ferner sei noch erwähnt, daß das neue in der Originalarbeit angegebene Verseifungsäquivalent für beide Fette ungewöhnlich ist, besonders auch das Verhältnis beider Verseifungsäquivalente zueinander.

Es dürfte daher wohl zweckmäßig sein, zunächst die in dieser Veröffentlichung befindlichen Angaben über die Zusammensetzung des Cocosnuß- und Palmkernöles mit Vorsicht aufzunehmen.

[A. 62.]

1) Analyst 39, 78; Angew. Chem, 28, II, 99 [1815].

2) Z. öff. Chem. 19, 88, [1913]. 